\title{
THE WHITE DWARF MASS AND ORBITAL PERIOD DISTRIBUTIONS IN ZERO-AGE CATACLYSMIC VARIABLES
}

\author{
M. Politano (1) and R.F. Webbink (2)
}

(1) Department of Physics, Arizona State University, U.S.A.

(2) Department of Astronomy, University of Illinois, U.S.A.

A zero-age cataclysmic variable (ZACV) we define as a binary system at the onset of interaction as a cataclysmic variable. We present here the results of calculations of the distributions of white dwarf masses and of orbital periods in ZACVs, due to binaries present in a stellar population which has undergone continuous, constant star formation for $10^{10}$ years. These results differ from previous work (Politano and Webbink 1988) in that an improved criterion for stability against rapid mass transfer by the secondary to the white dwarf has been used. A brief outline of the method and key assumptions made in this calculation is given in Politano and Webbink (1988).

The white dwarf mass distribution of ZACVs (shown in Figure 1) contains two components: systems with helium white dwarfs and systems with C-O white dwarfs. Systems with helium white dwarfs comprise slightly greater than $50 \%$ of all ZACVs. The helium white dwarfs have masses which range from 0.27 to 0.46 solar mass. The $\mathrm{C}-\mathrm{O}$ white dwarfs have masses which range from 0.54 solar mass up to the Chandrasekhar mass. (Note: systems with $\mathrm{O}-\mathrm{Ne}-\mathrm{Mg}$ white dwarfs are not distinguished from systems with $\mathrm{C}-\mathrm{O}$ white dwarfs in this calculation. Presumably, these $\mathrm{O}-\mathrm{Ne}-\mathrm{Mg}$ systems comprise the upper end of the white dwarf mass distribution.)

The distribution of white dwarf masses in ZACVs is similar to, although steeper than, the distribution of masses in single white dwarfs for masses above approximately 0.7 solar mass. The distribution of white dwarf masses in ZACVs is not sufficient to account for the obscrved high white dwarf masses in CVs, emphasizing the possible importance of selection effects.

The distribution of orbital periods (Figure 2) is comprised of five main subsets of ZACVs: 1) ultrashort-period systems containing degenerate (hydrogen) secondaries, 2) short-period systems containing helium white dwarfs formed below the period gap, 3) systems containing $\mathrm{C}-\mathrm{O}$ white dwarfs whose secondaries are convectively stable against rapid mass transfer to the white dwarf, 4) systems containing $\mathrm{C}-\mathrm{O}$ white dwarfs whose secondaries are radiatively stable against rapid mass transfer to the white dwarf, and 5) long-period systems containing evolved secondaries. Minima occur in the orbital period distribution between approximately 2 and 3 hours, arising from the discontinuity in cores masses between systems with helium white dwarfs and systems with C-O white dwarfs, and between approximately 3 and 5 hours, arising from the exclusion of systems with secondaries that are unstable to rapid mass transfer to the white dwarf.

We are also better able to identify the masses and orbital periods of the CV progenitors. We find that the masses of the primaries in the zero-age main sequence (ZAMS) progenitor binaries range from 0.95 solar mass to approximately 13 solar masses. The orbital periods of the ZAMS progenitor binaries range from 12 days to 1300 days.

This research was supported in part by NSF grants AST 86-16992 and AST 85-16173.

\section{References:}

Politano M. and Webbink R.F. 1988, in IAU Colloquium 114, White Dwarfs, ed. G. Wegner, (Springer-Verlag: Berlin), p. 440. 
WHITE DWARF MASS DISTRIBUTION

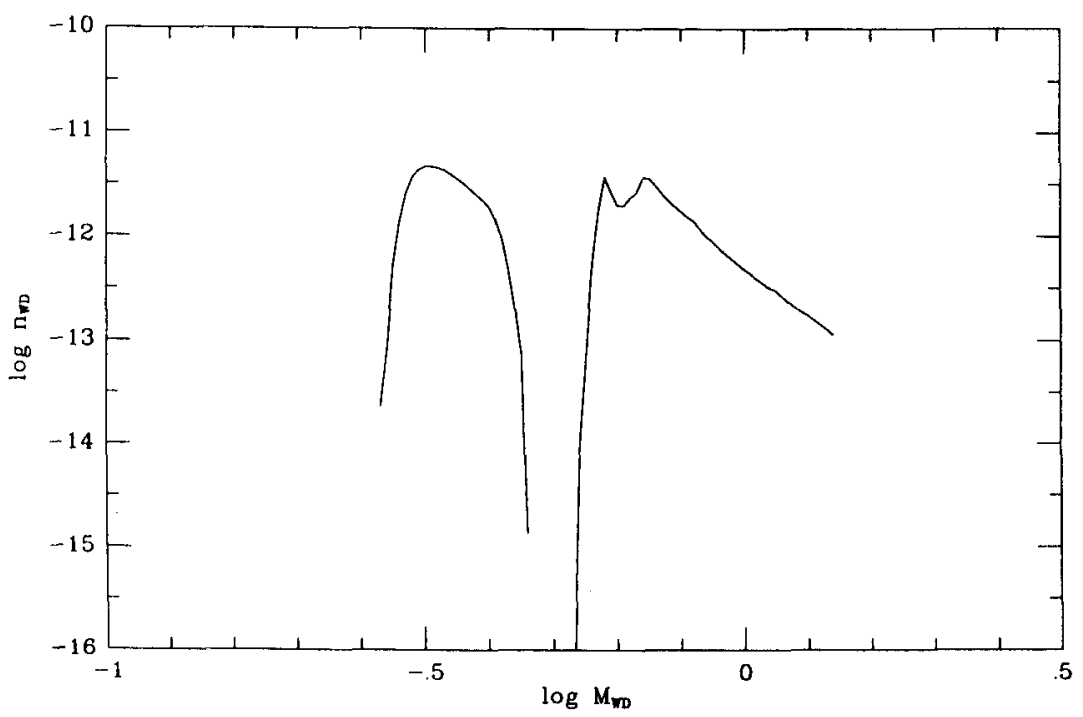

Figure 1: The distribution of the white dwarf masses in ZACVs due to all binaries formed within the last $10^{10}$ years. $M_{W d}$ is the mass of the white dwarf in solar masses and $n_{w d}$ is the number of CVs forming per year per log white dwarf mass per unit area of the Galactic plane (in $\mathrm{pc}^{2}$ ).

ORBITAL PERIOD DISTRIBUTION

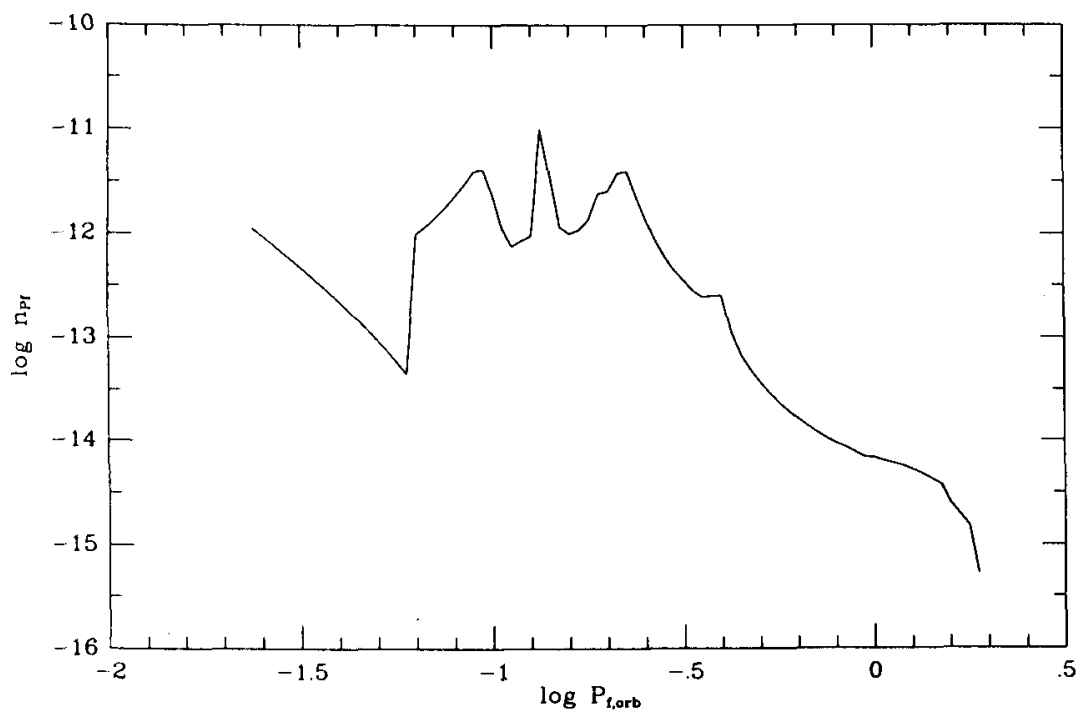

Figure 2: The distribution of the orbital periods in ZACVs due to all binaries formed within the last $10^{10}$ years. $P_{f}$,orb is the orbital period in days and $n_{P f}$ is the number of CVs forming per year per log orbital period per unit area of the Galactic plane (in $\mathrm{pc}^{2}$ ). 